\title{
Transcellular transport of Three kinds of diester Aconitineacross human intestinal Caco-2 cells
}

\author{
Fei Xiao Zhang, Dan Dan Liu, Xing Zi Ma, Xin Miao, Gang Li \\ Inner Mongolia Medical University, Inner Mongolia Hohhot City China, China
}

Objective:To study the transport behavior in Caco-2 cells of the radix aconitine,mesaconitine and hypaconitine, discusses three kinds of aconitine transport mode.

Methods:The drug concentration was determined by MTT method. The indexes were cumulative transfer amount and the apparent permeability coefficient Papp.The UPLC/Q Exactive MS method was used to detect the composition,study three aconitine that initial concentration was $100 \mathrm{ug} / \mathrm{ml}$ in both apical-to-basolateral and the reverse direction transport behavior of Caco-2cells on the model.Results:Three kinds of aconitine cumulative transfer amount and incubation time were positive correlation. The cumulative transfer amount of AP-BL side was about 2500ng.BL-AP side cumulative translocation amount was about 6000ng.within0-150min.And the three kinds of aconitine Papp value had no significant difference.The efflux of $\mathrm{AC}$ was more than two-fold that in the opposite direction.Conclusion:Results indicated that aconitine,mesaconitine and hypaconitine were mainly passive transporters,and might be involved in efflux protein, and the absorption was good. 